\title{
"Vein Involvement During Pancreaticoduodenectomy: Is There a Need for Redefinition of Borderline Resectable Disease": a Commentary on the Article Published by Kelly et al. in the Journal of Gastrointestinal Surgery 17:1209 (2013)
}

\author{
Sanjay S. Reddy • John P. Hoffman
}

Received: 1 August 2013 / Accepted: 10 February 2014 / Published online: 25 February 2014

(C) 2014 The Society for Surgery of the Alimentary Tract

\section{Dear Editors,}

We would like to make several comments on the recently published article entitled, Vein involvement during pancreaticoduodenectomy: is there a need for redefinition of "borderline resectable disease"? 1

Although no "final" definitions have been made regarding what defines a borderline resectable cancer, between the MD Anderson, SSO/AHPBA/SSAT, and NCCN consensus, attempts at a uniform classification have been tried. Regardless of the classification system used, these are defined by proper cross-sectional imaging. How many of these patients with venous resection had venous involvement noted on preoperative imaging? If patients did not have venous impingement by imaging, then they should not be classed as borderline resectable (as all definitions include distortion or impingement of the vein). Without accurately knowing the presence or degree of borderline resectability, it is premature to suggest that PV/ SMV involvement be dropped as a criterion for borderline resectability.

Chun et al. ${ }^{2}$ showed a clear difference in survival in the groups with unilateral portal vein and superior mesenteric vein shifts when treated with neoadjuvant therapy, as compared to a similar group given surgery first.

Secondly, the degree of histologic venous involvement was not characterized in the current study. Depth of venous involvement correlates with imaging characteristics and prognosis. Fukuda et al. ${ }^{3}$ showed that the depth of vein invasion significantly alters survival after curative resection. Deeper wall invasion into the tunica media or intima was associated with a poorer 1-year survival rate, similar to that of patients undergoing non-curative resection. The present study suggests that on multivariate analysis, vein involvement was not predictive of disease-free or overall survival, but how many of these patients had intimal or medial involvement? Since histologic data is not reported, these patients could all have had no actual histologic venous involvement.

The complete answer to this issue would be best obtained by a phase III study of neoadjuvant therapy versus upfront surgery in those with superior mesenteric vein/portal vein compression without occlusion. However, any study examining the issue should surely have both imaging and histologic descriptions of the patients studied.

\section{References}

1. Kelly et al. Vein Involvement During Pancreaticoduodenectomy: Is there a need for Redefinition of "Borderline Resectable Disease"? Journal of Gastrointestinal Surgery 17:1209 (2013)

2. Chun et al. Defining venous involvement in borderline resectable pancreatic cancer. Annals of Surgical Oncology 17:2832 (2010)

3. Fukuda et al. Significance of the Depth of Portal Vein Wall Invasion After Curative Resection for Pancreatic Adenocarcinoma. Archives of Surgery 142:172 (2007) 